\title{
Multiple Comparison Procedures for Determining the Optimal Complexity of a Model
}

\author{
Pedro L. Galindo, Joaquín Pizarro-Junquera, and Elisa Guerrero \\ Universidad de Cádiz - CASEM \\ Dpto. Lenguajes y Sistemas Informáticos \\ Grupo "Sistemas Inteligentes de Computación" \\ Polígono Río San Pedro, s/n \\ 11510 Puerto Real (CADIZ) \\ SPAIN \\ \{pedro.galindo, joaquin.pizarro,elisa.guerrero\}@uca.es
}

\begin{abstract}
We aim to determine which of a set of competing models is statistically best, that is, on average. A way to define "on average" is to consider the performance of these algorithms averaged over all the training sets that might be drawn from the underlying distribution. When comparing more than two means, an ANOVA F-test tells you whether the means are significantly different, but it does not tell you which means differ from each other. A simple approach is to test each possible difference by a paired t-test. However, the probability of making at least one type I error increases with the number of tests made. Multiple comparison procedures provide different solutions. We discuss these techniques and apply the well known Bonferroni method in order to determine the optimal degree in polynomial fitting and the optimal number of hidden neurons in feedforward neural networks.
\end{abstract}

\section{Introduction}

We consider the general problem of determining which of a set of competing models is best. Although there is active debate within the research community regarding the exact meaning of "best", statistical approaches are reasonable. Statistical approach to model selection tries to find which model is better on average. A way to define "on average" is to consider the performance of a given algorithm averaged over all the training sets that might be drawn from the underlying distribution. In a real situation, the underlying distribution is unknown, and we only have a finite size sample to work with.

In the following sections, we first describe the design of a randomized data collecting procedure required to control the different sources of variation. This design will allow us to generate several training sets following the underlying distribution, taking into account the different sources of variation that could exist [3]. After collecting the data, our goal will be to make inferences about $\mathrm{k}$ population means. $\mathrm{Al}$ 
though the ANOVA test allows us to reject the null hypothesis that the groups' means are all equal, it does not pinpoint where the significant differences lie. Multiple $t$ tests are not appropriate because the probability of a type I error increases with the number of comparisons made [5]. Statistical methods to compare three or more means while controlling the probability of making at least one type I error are called multiple comparison procedures. We briefly discuss these methods, including Fisher's LSD, Tukey's HSD, Bonferroni, Newman-Keuls, Duncan and Scheffé procedures and comment its potential advantages.

We will show how it is possible to apply these techniques to model selection through two examples. First, this model selection strategy is applied to determine the optimal degree in polynomial fitting. Results show that the optimal degree obtained is, in fact, the degree of the polynomial from which data are generated. Second, the same procedure is applied to determine the number of hidden neurons in feedforward networks. Obviosly, in this case, we can not validate the results.

\section{Design of the Experiment}

In order to compare different models, we must guarantee the independence of the results by controlling the sources of variation which affect the behaviour of the models. Dietterich [4] has analysed the sources of variation which a good statistical test should control. These sources of variation are controlled as follows:

- Variation resulting from the choice of the training and test data sets. On any particular randomly drawn test and training data sets, one model may outperform another. Given that we are studying how the models behave in average, we should repeat the estimation of the error over different training and test sets, and determine if any mean of errors is significantly smaller than the others. In order to compare different means, we recommend at least 30 measures to reduce the standard error for the comparisons.

- Variation resulting from the size of the test and training data sets. The performance of two different models changes smoothly with changes in the size of the training set. If a large amount of data is available, it is possible to set some of it aside to serve as a test set for evaluating the performance of the treatment. However, in most situations, the amount of data is limited and the use of all of it as input set is needed. Cross-Validation and Bootstrap procedures are the most common forms of resampling. However resampling means that each pair of training sets shares a high ratio of the samples. This problem of overlapping can be solved by using two-fold cross-validation, which involves the partition of the data set into two disjoints sets [8], training and test sets, of the same size.

- Internal randomess in the estimation of the model parameters. If the estimation of parameters is analytical and its determination is unique, this step can be omitted because there is no internal randomness. However, in an iterative approach the results depend critically on the starting state. Most of the iterative procedures suffer from internal randomness due to the initialisation of the parameter set to small random values. This parameter set depends on the model complexity, so it is dif- 
ferent in value and number for each model. Hence, to control this source of variation, several starting states are taken for each training data set. We focus our study in the model behaviour on average, so the extreme cases (the minimum and the maximum error estimates) are excluded and the mean error of the remaining results is considered to be the actual error of the model.

The complete strategy repeats a similar process 30 times: random splitting of data into a pair of equal sized portions and two-fold cross-validation for the estimation of the error for each model. The whole process is summarized as follows:

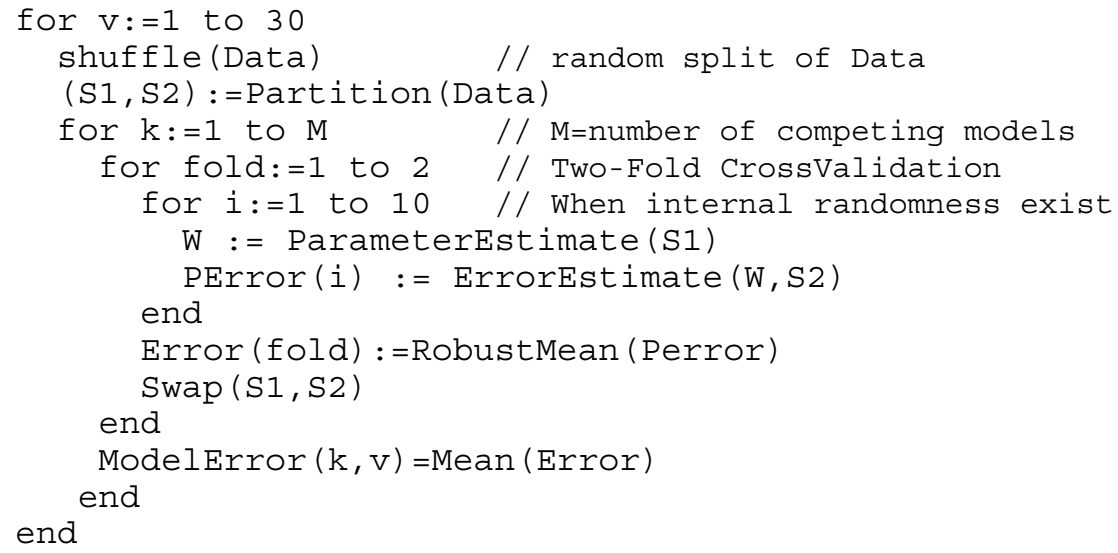

\section{Testing for Differences among Means in Groups}

Once we have obtained a set of error measures for each model that controls all the possible sources of variation of the experiment, we should compare them. First, we consider the problem of determining whether the means of error measures can be statistically considered equal or different. We study the assumptions that should be verified in order to make any valid inference. Second, we consider a more difficult problem: given that we know that error means are not equal, which of them is significantly smaller than the others?

\subsection{Are the Means Equal?}

As a first step, we may consider the use of a $t$-test to assess the means equality of two populations. But, if we are interested in testing whether the means of more than two populations are not significantly different, we must use a procedure called the analysis of variance (ANOVA)[7].

Analysis of variance is a parametric technique that tests the null hypothesis that the population means are equal to each other. However, in order to make conclusions about population means, several assumptions should be taken into account: 
- All $k$ population probability distributions should be normal. While this assumption is not relevant with large sample sizes, it is important with small sample sizes (specially with unequal sample sizes). This assumption has been tested using the method of Kolmogorov-Smirnov and we have always found that the distribution of results follows a Gaussian curve.

- $\quad$ The $k$ population variances should be equal. This assumption is not meaningful when all the models have the same (or almost the same) number of error subjects, but it is very important when this number differs. In our method the number of error measures is the same in all the models.

- The samples from each population should be random and independent. This assumption depends strongly on the design of the experiment. As the sources of variation have been taken into account, we assume random and independent data samples. Strictly speaking, the independence of the samples is not verified in our design, given that different results have been obtained from splitting randomly the finite sized available data. However, considering pairwise comparisons, the violation of this assumption is secondarily considered.

When assumptions for analyzing collected data from a completely randomized design are violated, any inferences derived from the ANOVA are suspect. An alternative technique to use in this situation is the nonparametric Kruskal-Wallis test.

\subsection{Which Means Are Equal?}

When comparing more than two means, an ANOVA F-test tells you whether the means are significantly different, but it does not tell you which means differ from each other. The first idea that comes to mind is to test each possible difference by a paired t-test. However, this approach increases the probability of making at least one type I error with the number of tests made. Statistical methods to compare three or more means while controlling the probability of making at least one type I error are called multiple comparison procedures.

\section{Multiple Comparison Procedures}

Multiple comparison procedures compare the average effects of three or more treatments to decide which treatments are better, which ones are worse, and by how much, while controlling the probability of making an incorrect decision. A wide range of multiple comparison procedures is commonly present in the literature 6 .

The Fisher's Least Significant Differences(LSD) procedure begins with a one-way analysis of variance. Only when the overall F-ratio is statistically significant we carry out all possible t-tests. Some authors refer to this procedure as Fisher's Protected LSD to emphasize the protection provided by the F-ratio.

Tukey's Honestly Significant Differences(HSD) follows the path of Student, determining the distribution of the largest $t$ statistic when many groups are compared and 
there are no underlying differences. It is a test specifically designed for pairwise comparisons when the sample sizes are equal. Tukey and Kramer independently propose a modification for unequal cell sizes. Two means are considered significantly different by the Tukey-Kramer criterion if

$$
\left|t_{i j}\right| \geq q(\alpha ; k ; v)
$$

where $\mathrm{q}(\alpha ; \mathrm{k} ; \mathrm{v})$ is the $\alpha$-level critical value of a studentized range distribution of $k$ independent normal random variables with $v$ degrees of freedom.

Bonferroni[2] is a well known and easy to apply follow-up analysis of the Anova F-test. This procedure adjusts the observed significance level based on the number of comparisons we are making. This technique compares the difference between two treatment means to a critical difference. This difference depends on the number of observations in each treatment, the significance level, the variability unexplained by the differences between the sample means, and the total number of treatments to be compared. If the difference between the sample means exceeds the critical difference, there is sufficient evidence to conclude that the population means differ. Bonferroni $t$ test declares two means to be signicantly different if:

$$
\left|t_{i j}\right| \geq t(\varepsilon ; v)
$$

where

$$
\varepsilon=\frac{2 \alpha}{\mathrm{k}(\mathrm{k}-1)}
$$

for comparisons of $k$ means.

The Student-Newman-Keuls (SNK) procedure is an attempt to compromise between LSD and HSD. Like the Tukey HSD it is based on a studentized range distribution. This procedure is more powerful than the Tukey HSD and is better at controlling the experimentwise error rate (EER). However it is less often used, mainly for two reasons. First, it cannot be used to construct confidence intervals for differences between means. Second, there are patterns of population means which lead to an inflated EER.

Duncan's method looks much like the SNK procedure and gives many more significant differences. It is only very slightly more conservative than Fisher's LSD, and, in practice, in the majority of the cases they lead to the same conclusions.

A technique slightly less conservative than Bonferroni is the Sidak test given by

$$
\left|t_{\mathrm{ij}}\right| \geq \mathrm{t}(\varepsilon ; v)
$$

where

$$
\varepsilon=1-(1-\alpha)^{\frac{2}{k(k-1)}}
$$

for comparisons of $k$ means. 
Scheffé proposes another method to control the maximum error rate under any complete or partial null hypothesis. Two means are declared significantly different if

$$
\left|t_{i j}\right| \geq \sqrt{(k-1) \mathrm{F}(\alpha ; k-1 ; v)}
$$

where $\mathrm{F}(\alpha ; k-1 ; v)$ is the $\alpha$ level critical value of an $\mathrm{F}$ distribution with $\mathrm{k}-1$ numerator degrees of freedom and $v$ denominator degrees of freedom. Scheffé test never declares a contrast significant if the overall F-test is nonsignificant.

Scheffé method may be more powerful than the Bonferroni or Sidak methods if the number of comparisons is large relative to the number of means. The Tukey-Cramer method is more powerful than the Bonferroni, Sidak or Scheffé methods for pairwise comparisons.

As a conclusion, we maintain that there is no "correct" procedure to use. The various procedures trade off power for control of the EER in different ways. Most researchers believe that the Duncan's and Fisher's LSD procedures result in too high an EER and should not be used. If you want to be sure that you have controlled the EER, then the Tukey HSD should be used at the expense of a lower power. In practice, it is advisable to avoid conducting multiple comparisons of a small number of treatment means when the corresponding ANOVA F test is nonsignificant; otherwise, confusing and contradictory results may occur. Finally, we should remember that failure to reject the hypothesis that two or more means are equal should not lead to conclude that the population means are, in fact, equal. Failure to reject the null hypothesis implies only that the differences between population means, if any, is not large enough to be detected with the given sample size.

\section{Simulation Results}

In this section we provide two examples of model order selection by using the Bonferroni multiple comparison procedure. Given a model selection problem, we proceed as follows:

1. Select an error criterion

2. Generate 30 values of error for each model as specified in section 2

3. Select the desired overall confidence level : $\alpha=0.1$

4. Use ANOVA F-test to determine whether the means error are significantly different from each other.

5. For each model, determine the set of models not significantly different by Bonferroni method.

6. If the groups are not overlapped, select the model with the least error, and select the most simple model in its group. Otherwise, select the model with the least error.

\subsection{Determining the Degree of Polynomial Fitting}

Let us consider the problem of finding the degree $\mathrm{N}$ of a polynomial $\mathrm{P}(\mathrm{x})$ that better fits a set of data in a least squared sense. Figure 1 shows the experimental curve and a 
set of 160 data points generated by adding gaussian noise which will be used in the experiment. 80 data points will be used to determine the coefficients, and 80 will be used to calculate the RMS error. The only aspect of the polynomials which remains to be specified is the degree(M), and so we use a set of polynomials with degree ranging from 1 to 10 .

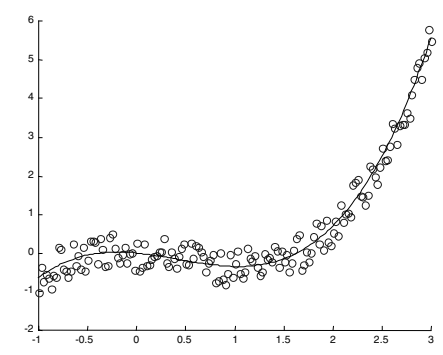

Figure 1: Experimental curve and data points for polynomial fitting. The experimental polynomial is $\mathrm{P}(\mathrm{x})=0.4 \mathrm{x}^{3}-0.5 \mathrm{x}^{2}-0.25 \mathrm{x} \quad \mathrm{x} \in[-13]$.

As we explained above, 30 RMS errors for each polynomial have been generated. We used ANOVA F-test to determine whether the means RMSE are significantly different form each other and Bonferroni method to determine whether the observed differences in the sample means imply that differences exist among the accuracy of the competing polynomials. The overall confidence level is fixed to 0.1

Table 1 shows the results obtained in this case. This table shows the polynomial degree, the RMSE error and the set of polynomial degree not significantly different. Two polynomials are not significantly different if the difference between its means is less than the critical value computed as 0.02256 . In this case, there are three groups. Polynomials from degree 3 to 10 form a not significantly different RMSE group and a polynomial of degree 3 is selected (Occam's Razor criterion [1]).

Table 1: Simulation results (160 data points)

\begin{tabular}{|c|c|c|}
\hline $\begin{array}{l}\text { Polynomial } \\
\text { degree }\end{array}$ & RMSE & $\begin{array}{c}\text { Polynomial degrees } \\
\text { not significantly different }\end{array}$ \\
\hline 3 & 0.04261 & $\begin{array}{llllllll}3 & 4 & 5 & 6 & 7 & 8 & 9 & 10 \\
\end{array}$ \\
\hline 4 & 0.04340 & $\begin{array}{llllllll}3 & 4 & 5 & 6 & 7 & 8 & 9 & 10\end{array}$ \\
\hline 5 & 0.04406 & $\begin{array}{llllllll}3 & 4 & 5 & 6 & 7 & 8 & 9 & 10\end{array}$ \\
\hline 6 & 0.04519 & $\begin{array}{llllllll}3 & 4 & 5 & 6 & 7 & 8 & 9 & 10\end{array}$ \\
\hline 7 & 0.04543 & $\begin{array}{llllllll}3 & 4 & 5 & 6 & 7 & 8 & 9 & 10\end{array}$ \\
\hline 8 & 0.04655 & $\begin{array}{llllllll}3 & 4 & 5 & 6 & 7 & 8 & 9 & 10 \\
\end{array}$ \\
\hline 9 & 0.04777 & $\begin{array}{llllllll}3 & 4 & 5 & 6 & 7 & 8 & 9 & 10\end{array}$ \\
\hline 10 & 0.04903 & $\begin{array}{llllllll}3 & 4 & 5 & 6 & 7 & 8 & 9 & 10 \\
\end{array}$ \\
\hline 2 & 0.18750 & 2 \\
\hline 1 & 0.50280 & 1 \\
\hline
\end{tabular}


Table 2 shows the results when the size of data point is 40 . Two polynomial are not significantly different if the difference between its means is less than the critical value computed as 2.75873. In this case the groups are overlapped. Because variation among RMSE means are not significant, polynomial degree with the least RMSE means is selected. The model with degree 3 is selected.

Table 2: Simulation results (40 data points)

\begin{tabular}{|c|c|c|}
\hline $\begin{array}{c}\text { Polynomial } \\
\text { degree }\end{array}$ & RMSE & $\begin{array}{c}\text { Polynomial degrees } \\
\text { not significantly different }\end{array}$ \\
\hline 3 & 0.06426 & $\begin{array}{llllllllll}3 & 4 & 5 & 6 & 7 & 2 & 8 & 1 & 9\end{array}$ \\
\hline 4 & 0.07468 & $\begin{array}{lllllllll} & 4 & 5 & 6 & 7 & 2 & 8 & 1 & 9\end{array}$ \\
\hline 5 & 0.10979 & $\begin{array}{lllllllll}3 & 4 & 5 & 6 & 7 & 2 & 8 & 1 & 9\end{array}$ \\
\hline 6 & 0.11570 & $\begin{array}{lllllllll}3 & 4 & 5 & 6 & 7 & 2 & 8 & 1 & 9\end{array}$ \\
\hline 7 & 0.15173 & $\begin{array}{llllllllll} & 4 & 5 & 6 & 7 & 2 & 8 & 1 & 9\end{array}$ \\
\hline 2 & 0.28682 & $\begin{array}{llllllllll} & 4 & 5 & 6 & 7 & 2 & 8 & 1 & 9\end{array}$ \\
\hline 8 & 0.45635 & $\begin{array}{llllllllll}3 & 4 & 5 & 6 & 7 & 2 & 8 & 1 & 9 \\
\end{array}$ \\
\hline 1 & 0.78130 & $\begin{array}{llllllllll}3 & 4 & 5 & 6 & 7 & 2 & 8 & 1 & 9 & 10 \\
\end{array}$ \\
\hline 9 & 0.97943 & $\begin{array}{llllllllll}3 & 4 & 5 & 6 & 7 & 2 & 8 & 1 & 9 & 10 \\
\end{array}$ \\
\hline 10 & 3.32416 & 19910 \\
\hline
\end{tabular}

\subsection{Determining the Number of Hidden Neurons in Multiplayer Perceptrons}

Let us now consider the problem of determining the number of hidden units in a feed-forward neural network in a classification task. Let us define a data set where each input vector has been labelled as belonging to one of two classes $C_{1}$ and $C_{2}$. Figure 2 shows the input patterns. In the simulation study, we consider multi-layer perceptrons having two layers of weights with full connectivity between adjacent layers. One linear output unit, $\mathrm{M}$ hidden units and no direct input-output connections. The only aspect of the architecture that remains to be specified is the number $\mathrm{M}$ of hidden units, and so we train a set of networks (models) having a range of values of $\mathrm{M}$.

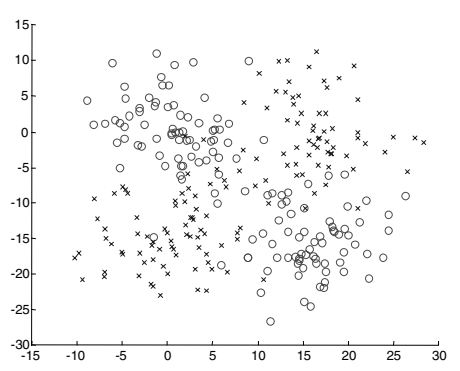

Figure 2. Sample data distribution. The sample size is $\mathrm{N}_{1}=280$ data of the class $C_{1}$ and $\mathrm{N}_{2}=140$ of the class $C_{2}$. 
Table 3 shows the simulation results in this case. Two models are in the same group if the difference between its means is less than the critical value, 0.02212 . Thus, from the group of models with less error mean (10 hidden units) the model with 4 hidden units is selected by Occam's Razor criterion. If the number of models to be compared increases, results show that four hidden units is a good selection; that is, there is not a statistically significant difference among the error means of neural network architecture with four or more hidden units.

Table 3. Simulation Results (280 data points)

\begin{tabular}{|c|c|c|}
\hline $\begin{array}{c}\text { Hid- } \\
\text { den Units }\end{array}$ & $\begin{array}{l}\text { Error } \\
\text { Mean }\end{array}$ & $\begin{array}{c}\text { Models not } \\
\text { significantly different }\end{array}$ \\
\hline 7 & 0.13790 & $\begin{array}{lllllll}7 & 5 & 8 & 6 & 10 & 9 & 4\end{array}$ \\
\hline 5 & 0.13995 & $\begin{array}{lllllll}7 & 5 & 8 & 6 & 10 & 9 & 4\end{array}$ \\
\hline 8 & 0.13995 & $\begin{array}{lllllll}7 & 5 & 8 & 6 & 10 & 9 & 4\end{array}$ \\
\hline 6 & 0.14033 & $\begin{array}{lllllll}7 & 5 & 8 & 6 & 10 & 9 & 4\end{array}$ \\
\hline 10 & 0.14214 & $\begin{array}{lllllll}7 & 5 & 8 & 6 & 10 & 9 & 4\end{array}$ \\
\hline 9 & 0.14319 & $\begin{array}{lllllll}7 & 5 & 8 & 6 & 10 & 9 & 4\end{array}$ \\
\hline 4 & 0.14900 & $\begin{array}{lllllll}7 & 5 & 8 & 6 & 10 & 9 & 4 \\
\end{array}$ \\
\hline 3 & 0.18848 & 3 \\
\hline 2 & 0.31433 & 2 \\
\hline 1 & 0.35938 & 1 \\
\hline
\end{tabular}

Table 4 shows the results when the number of data points is 60 . In this case two models are in the same group if the difference between its means is less than 0.08818 . We can see that the groups are overlapped. This may be due to two main reasons: either we haven't enough data points or the training has been stopped too soon. Because variation among misclassification error means is not significant, the model with the least error, 5 hidden units, is selected.

Table 4: Simulation results (60 data points)

\begin{tabular}{|c|c|rrr|r|r|}
\hline $\begin{array}{c}\text { Hid- } \\
\text { den Units }\end{array}$ & $\begin{array}{c}\text { Error } \\
\text { Mean }\end{array}$ & \multicolumn{8}{|c|}{ Models not significantly } \\
different
\end{tabular}




\section{Conclusions}

We have proposed a model selection strategy based on multiple comparison procedures. ANOVA test can be applied to compare the population means and to determine the existence of significant differences among the competing models. However, the proper application of the ANOVA procedure requires certain assumptions to be satisfied. When the number of tests increases, the probability of making a type I error increases with the number of comparisons. Statistical methods to deal with this phenomenon are called multiple comparison procedures, since they can compare three or more means while controlling the probability of making at least one type 1 error. When this strategies are adequately applied to the error rates of a well designed experiment, the needed assumptions are verified, and it is possible to determine the optimal complexity of a given model, or even more, to determine which of a family of models fits better to a given problem. This result has been shown to be useful determining the optimal degree in a polynomial fitting and the optimal number of hidden units in feedforward networks. Future work will address more specific comparison procedures and its application to other neuronal models, like radial basis function networks.

\section{References}

1. Bishop, C. M.: Neural Network for Pattern Recognition. Clarendon Press- Oxford (1995)

2. Cobb, G.W.: Introduction to Design and Analysis of Experiments. Springer-Verlag New York (1998)

3. Dean, A., Voss, D.: Design and Analysis of Experiments. Springer Texts in Statistics. Springer-Verlag New York (1999)

4. Dietterich, T.G.: Aproximate Statistical Test for Comparing Supervised Classification Learning Algorithms. Neural Computation (1998), Vol. 10, no.7, 1895-1923

5. Feelders, A., Verkooijen, W.: On the Statistical Comparison of Inductive Learning Methods. Learning from Data Artificial Intelligence and Statistics V. Springer-Verlag, New York (1996) 271-279

6 Hsu, J.C.: Multiple Comparisons: Theory and Methods. Chapman \& Hall (1996)

7. Jobson, J.D.: Applied Multivariate Data Analysis. Springer Texts in Statistics, Vol 1. Springer-Verlag New York (1991)

8. Stone, M.: Cross-validatory Choice and Assesment of Statistical Prediction (with discussion). Journal of the Royal Statistical Society (1974), Series B, 36, 111-147 